\title{
STRESS AND DEFORMATION ANALYSIS OF THE METAL EXTRUSION PROCESS *
}

\author{
E.H. LEE and R.L. MALLETT \\ Stanford University, Stanford, Calif. 94305, USA \\ and \\ W.H. YANG \\ University of Michigan, Am Arbor, Mich. 48104, USA
}

Received August 1976

\begin{abstract}
A complete stress analysis of a metal-forming process is necessary in order to assess the onset of metal-forming defects such as the initiation of internal or surface cracks or the generation of residual stresses. This demands elasticplastic analysis. A program to evaluate complete stress distributions has been developed and applied to the extrusion process. Such solutions have not previously been obtained for general two- and three-dimensional problems encompassing the technologically important steady state processes. although these solutions are essential for the rational assessment of limits on process variables which will ensure a satisfactory metal-forming procedure. The stress fields obtained for the extrusion process exhibit features which are consistent with the known development of extrusion defects, such as the appearance of surface cracks.
\end{abstract}

\section{Introduction}

In order to develop a rational basis for determining the limitations on design and operational factors for metal-forming processes which will ensure a satisfactory product, it is necessary to evaluate the history of the stress and deformation distributions throughout the work-piece. Characteristics - termed metal-forming defects - which yield an unsatisfactory product include the generation of internal and surface fractures, the onset of local instabilities causing local strain peaks, and the production of high residual stresses. In many metal-forming processes, plastic strains of the order unity occur, and these completely dominate the elastic strains which are of the order the yield stress divided by the elastic modulus, which is of the order $10^{-3}$. Nevertheless, elastic strain increments, particularly on initial loading and unloading, play an essential role in determining the stress distribution. The criteria governing the initiation and growth of cracks are commonly based on the history of the stress in an element, which thus plays a major role in predicting the production of metal-forming defects. Residual stresses, of course, constitute the stress field after the work-piece has emerged from the process.

Consistent with the already stated influence of elastic strains in determining the stress field, the plastic-rigid theory (which neglects the elastic strains completely) can only predict stresses in the

\footnotetext{
* Presented at the 14th International Congress of Theoretical and Applied Mechanics, Delft, The Netherlands, August 30September 4, 1976. The contribution of E.H. Lee and R.L. Mallett was supported in part by the NSF's Material Research Laboratory Program through the Center for Materials Research at Stanford University and by Contract NSF-ENG-7 5-17376. E.H. Lee's contribution was in part generated while he held a John Simon Guggenheim Memorial Fellowship.
} 
regions of active plastic flow. The regions of "contained plastic flow" - in which increments of plastic strain are of the order of the elastic strain increments (since deformation there is constrained by the adjacent elastic material) - are considered to be rigid in the context of plasticrigid theory. Hence, no compatibility equations prescribing the consistency of the deformation field can be adjoined to the equilibrium equations; thus there are not enough equations available to determine the stress field. Elastic-plastic theory must therefore be utilized in the assessment of metal-forming processes aimed at prescribing conditions which will eliminate the generation of metal-forming defects and so are capable of predicting the formation of a satisfactory product

The structure of the equations governing elastic-plastic deformation calls for a quite different solution procedure from that for plastic-rigid theory. The incremental or rate structure of elasticplastic constitutive relations leads to relations of the type

$$
D_{i j}=\frac{1+v}{E} \dot{\sigma}_{i j}-\frac{v}{E} \delta_{i j} \dot{\sigma}_{k k}+\frac{9 \sigma_{i j}^{\prime} \sigma_{k l}^{\prime} \dot{\sigma}_{k l}}{4 h \bar{\sigma}^{2}}
$$

where

$$
D_{i j}=\frac{1}{2}\left(\frac{\partial v_{i}}{\partial x_{j}}+\frac{\partial v_{j}}{\partial x_{i}}\right)
$$

is the velocity strain, $E$ and $\nu$ are the elastic constants (Young's modulus and Poisson's ratio), $\sigma_{i j}$ is the stress tensor (the prime indicates the deviator or shear type components), $\bar{\sigma}$ is the current yield stress in tension, and $h$ is the tensile tangent modulus or strain-hardening coefficient associated with plastic strain in simple tension. The superposed dot indicates the appropriate rate derivative. The relation (1) corresponds to isotropic work hardening, and the variation of the strainhardening coefficient $h$ with strain or yield stress is determined from an experimental stress-strain curve in simple tension. In this formulation the velocity strain $D_{i j}$, expressed in terms of the velocity gradient $\partial v_{i} / \partial x_{j}$, comprises the rate-of-strain term. Stress analysis theory based on (1) thus involves the velocity distribution $v_{i}(\boldsymbol{x}, t)$ (where $\boldsymbol{x}$ is the current position vector of an element) and the stress-rate distribution $\dot{\sigma}_{i j}(x, t)$. Equations to determine these quantities are obtained from (1) and (2) together with equilibrium equations for the stress rates and boundary conditions. In terms of the velocity and stress-rate distributions at time $t$ the increments of stress and displacement in the time interval $(t, t+\Delta t)$ are obtained which provide the new configuration and stress at time $t+\Delta t$. This procedure is repeated sequentially to build up the complete stress and deformation history of the process.

This procedure differs fundamentally, for example from the well-known slip-line field theory of rigid-ideally plastic analysis of deformation in plane strain. With no elastic strain components to incorporate, stress-velocity relations arise, and direct determination of the stress field rather than the stress-rate field yields the solutions. Although this situation is modified if strain hardening is considered, when elastic strain components are neglected the rates of stress invariants onle appear in the equations which provide a simplification over elastic-plastic theory. The need to utilize stress-rate variables in the analysis of elastic-plastic theory introduces considerable complexity, since tensor-rate variables are more sensitive to convection effects and nonlinear geometrical influences than are the tensors themselves. This demands the use of extra care in satisfying the requirements of finite deformation continuum theory, and the influence of this on metal-forming analysis is summarized in the following section. 


\section{Basic elastic-plastic theory}

The stress-rate term in a constitutive relation must clearly be a derivative of the stress at a material particle, since it is the stress history at a particle which determines the deformation there. Rice pointed out [1] that the component of this rate associated with the motion of the material particle - the convected component - is of the same order as that due to the partial derivative of the stress at a fixed position in space when the tangent modulus associated with the constitutive relation is of the order of the stress. This is the case for the strain-hardening coefficient for many metals in plastic flow. This contrasts with the situation in classical elasticity for which the ratios of elastic moduli to stress are about $10^{3}$. Thus, in classical elasticity, convected terms are insignificant, and in the analysis there is no need to differentiate between the deformed and undeformed configurations.

Thus, for many plasticity problems, convected terms must be included in the analysis, and this involves extending the classical plasticity theory and variational principles in order to have a correct formulation. Such a generalized theory was developed by Hill [2] in studying the uniqueness of elastic-plastic solutions, bifurcation of solutions and the stability problem. For such studies meticulous care is needed in formulating rate definitions and the influence of noninfinitesimal displacements in order to achieve a correct analysis. It turns out that for general metal-forming problems the same care is also needed. A means of avoiding these difficulties in overt form is to express the deformation equations in terms of the reference geometry - for example the initial configuration of the body if Lagrange coordinates are used. This approach has been formulated for finite element calculations by McMeeking and Rice [3]. An analysis of this development has been given in [4], and our finite element elastic-plastic code has been modified accordingly. The advantage of using the reference configuration to supply the position variables with which to express the equations of deformation is that the reference configuration remains fixed so that partial derivatives with respect to time refer to variations at a specified material point, and variational principles comprise integrals over a fixed volume with no boundary motions.

Whereas referential variables are convenient for expressing the equations of motion, the laws of plasticity are incremental in nature, or of flow type, involving stress and strain rates, as in (1), defined relative to the current geometry. Experimentally, the plasticity laws of the yield condition and the flow relation involve true stress and increments of natural strain, both defined with respect to the current configuration. Corresponding expressions based on the reference geometry would be more involved. In order to avoid this complexity, the configuration at time $t$ is taken as the reference configuration for deformation increments in the time interval $(t, t+\Delta t)$. In this way we can take advantage of the use of not only referential coordinates for the equations of motion but also current coordinates for the plasticity laws. The details of this structure are presented in [4] and are incorporated in our computing program. Included in this structure is the correct influence of the rate of rotation of material elements. It turns out that this has the same order of influence on the theory as do the convected terms, and use of the spin-invariant Jaumann derivative for the rate of stress expression in (1) yields the correct equations of motion in conjunction with the classical form of plasticity law.

As developed for treating the elastic-plastic stability problem, the boundary conditions on the surface of the body were limited to prescribed particle velocities on part of the boundary and to a prescribed rate of traction based on the reference geometry on the remaining boundary surface. The sliding of the work-piece over a die surface present a more general boundary value problem 
prescription since neither velocity nor traction are independently prescribed. A modification of the previously developed variational principle is used to generate the finite element relations which incorporate this condition for smooth boundaries and is given in the following section.

\section{Boundary conditions on smooth die and driving piston surfaces}

The boundary conditions stated in [2] and [3] are prescribed nominal traction rate over part of the surface in the reference configuration and prescribed velocity over the rest. In this, our first solution of an extrusion problem, we shall consider frictionless curved dies and a frictionless driving piston for the direct extrusion of a strip in plane strain. This calls for mixed boundary conditions of zero shear traction at the metal-die interface and zero normal velocity component. For the driving piston the shear traction is zero and the normal velocity component is prescribed. The unknown normal tractions and tangential velocities are to be determined. We proceed as described in the previous section by sequentially building on the already evaluated solution at time $t$ by adding increments of stress and displacement corresponding to the time increment $\Delta t$. These are determined by a finite element analysis for the stress rates and velocities. A modification of the variational principle given by Hill [2] is needed to obtain the finite element formulation with the modified boundary conditions. The principle governs the unsymmetric nominal stress rate (or Piola-Kirchhoff I stress rate) $\dot{s}_{i j}$ and involves integration over the reference state, which is taken to be the configuration at time $t$ for the determination of stress rates and velocities at that instant. These are then used to determine the solution at time $t+\Delta t$, with $\Delta t$ sufficiently small for firstorder increments to be accurate:

$$
\boldsymbol{\sigma}(t+\Delta t)=\boldsymbol{\sigma}(t)+\dot{\sigma} \Delta t, \quad \boldsymbol{x}(t+\Delta t)=x(t)+v \Delta t,
$$

where $\sigma$ is the Cauchy or true stress, $\boldsymbol{x}$ is the position coordinate, and $v$ is the velocity.

Elastic-plastic theory determines a rate potential form for the nominal stress rate $\dot{s}_{i j}[2-4]$ :

$$
\dot{s}_{i j}=\partial E / \partial\left(\partial v_{j} / \partial x_{i}\right)
$$

where $E$ is a known quadratic rate potential function. The velocity and stress rate distributions can then be determined from a variational principle for the velocity field:

$$
\int_{V} \dot{s}_{i j} \delta\left(\partial v_{j} / \partial x_{i}\right) \mathrm{d} V-\int_{S} \dot{F}_{j} \delta v_{j} \mathrm{~d} S=0,
$$

the $\dot{s}_{i j}$ being expressed by (3); $V$ is the volume and $S$ the surface of the body at time $t$. This principle differs from that used in [2] since the nominal traction rate $\dot{F}_{j}$ is not necessarily prescribed when $\delta v_{j} \neq 0$ but must be determined; hence the $\delta$ cannot apply to the whole surface integral in the last term of (4).

By the usual divergence theorem manipulation eq. (4) can be shown to be equivalent to the equilibrium equation for stress rates

$$
\partial \dot{s}_{i j} / \partial x_{i}=0
$$


and the traction rate boundary condition

$$
\dot{F}_{j}=n_{i} \dot{s}_{i j}
$$

where $n_{i}$ is the unit surface normal at time $t$. Thus, if we select the unknown components of $\dot{F}_{j}$ to satisfy (6), the variational principle will yield the required solution determining stresses in static equilibrium.

For convenience, consider tangential and normal axes $\left(x_{1^{\prime}}, x_{2^{\prime}}\right)$ at the boundaries as shown in fig. 1 and express $\dot{s}_{i j}$ in terms of the Kirchhoff stress $\tau_{i j}[3,4]$ :

$$
\dot{s}_{i j}=\mathcal{D}^{c} \tau_{i j} / D t-\left(\sigma_{i k} D_{j k}+\sigma_{k j} D_{i k}\right)+\sigma_{i k} \partial v_{j} / \partial x_{k},
$$

where the first term on the right-hand side is the Jaumann derivative of the Kirchhoff stress expressed in Cartesian coordinates, and $\boldsymbol{D}$ is the velocity strain

$$
D_{i j}=\frac{1}{2}\left(\partial v_{i} / \partial x_{j}+\partial v_{j} / \partial x_{i}\right)
$$

Then at the boundary interfaces with the fixed smooth die (fig. 1a) and smooth driving piston with prescribed driving velocity $v_{0}$ (fig. $1 \mathrm{~b}$ ), $\delta v_{2^{\prime}}=0$ to avoid separation and $\tau_{1^{\prime} 2^{\prime}}=0$ because there is no friction and $[2-4]$

$$
\tau_{i j}=J \sigma_{i j},
$$

where $J$ is the Jacobian density ratio $\rho_{0} / \rho$. Moreover

$$
\mathcal{D}^{c} \tau_{1^{\prime} 2^{\prime}} / \mathcal{D} t=0
$$

since $\tau_{1^{\prime} 2^{\prime}}=0$ and the rotation of the boundary axes $\left(x_{1^{\prime}}, x_{2^{\prime}}\right)$ following a particle is the same as the average rotation involved in the Jaumann derivative. This is so because the boundary surface

a.

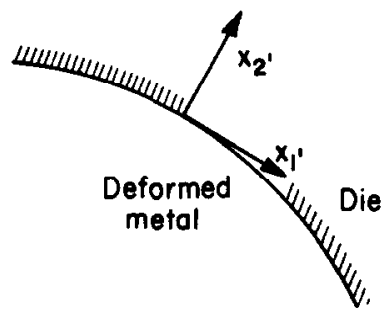

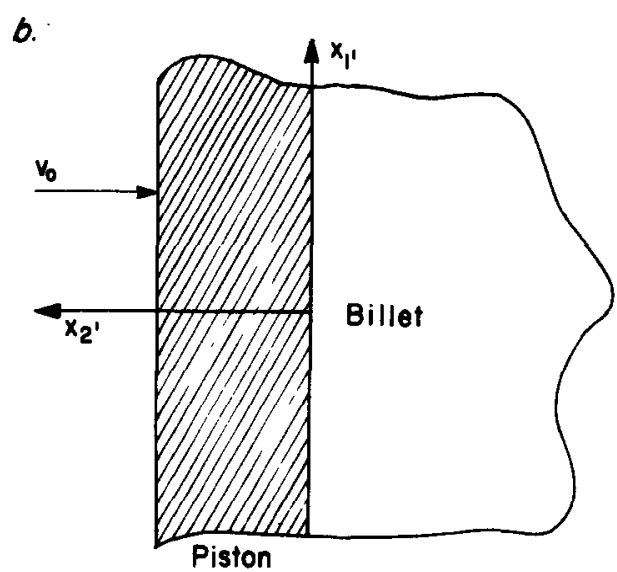

Fig. 1. (a) The die interface geometry, (b) the piston billet interface geometry. 
has been a principal direction of stress, and hence of strain rate, throughout the motion, which incidentally causes $D_{1^{\prime} 2}$, to be zero; thus

$$
n_{i^{\prime}}=(0,1)
$$

so that the contribution to the surface integral in (4) is

$$
\int_{S} \sigma_{2^{\prime} 2^{\prime}}\left(\partial v_{1^{\prime}} / \partial x_{2}\right) \delta v_{1}^{\prime} \mathrm{d} S
$$

Now $\partial v_{1^{\prime}} / \partial x_{2}$, is the angular velocity of the boundary, since the normal is also a principal direction of strain rate, and (12) becomes

$$
\int_{S} \sigma_{2^{\prime} 2^{\prime}}, \kappa v_{1}, \delta v_{1^{\prime}} \mathrm{d} S
$$

where $\kappa$ is the curvature of the boundary. The stress is known for each step of the calculation determining increments in the time intervals $(t, t+\Delta t)$ so that (13) introduces into the variational expression a term that is bilinear in the unknown $v_{1^{\prime}}$ and the known $\sigma_{2^{\prime} 2^{\prime}}$. Combined with the volume integral terms from (4), this modifies the coefficients in the equations for velocities and retains matrix symmetry. This procedure eliminates the unknown boundary traction-rate terms. In effect this formulation introduces stress dependent stiffness terms in the composite incremental stiffness matrix. The possibility of terms of this type is mentioned in [3]. Nonzero contributions of this form arise for curved dies, but for the flat driving piston the curvature $\kappa$ and hence the contribution are zero.

In practice it is simpler to express the boundary condition (6) directly in terms of nodal forces in the finite element formulation, and this approach is mentioned in the following section.

\section{Finite element formulation}

The elastic-plastic theory used for the analysis of this extrusion problem involves isotropic work-hardening based on a measured tensile stress-strain curve. A section reduction to $75 \%$ of the initial cross-section is considered which leads to the development of strains of the order unity. Thus material nonlinearity is introduced through the stress-strain curve, and geometrical nonlinearity is introduced by the finite deformations that occur. These features added to the complexity of elastic-plastic analysis indicate that numerical evaluation of the solution is mandatory. A finite element approach has been adopted because of the convenience it provides for discretizing the analysis of a problem posed by a variational principle and for satisfying boundary conditions on curved boundaries.

A finite element mesh is selected on the initial configuration of the workpiece to be extruded, and the deformation of these elements and the stresses generated in them are evaluated throughout the motion. Thus a convected network is utilized which deforms with the material. The initial configuration of a quadrilateral net in the undeformed state and after extrusion are shown in fig. 2 . 

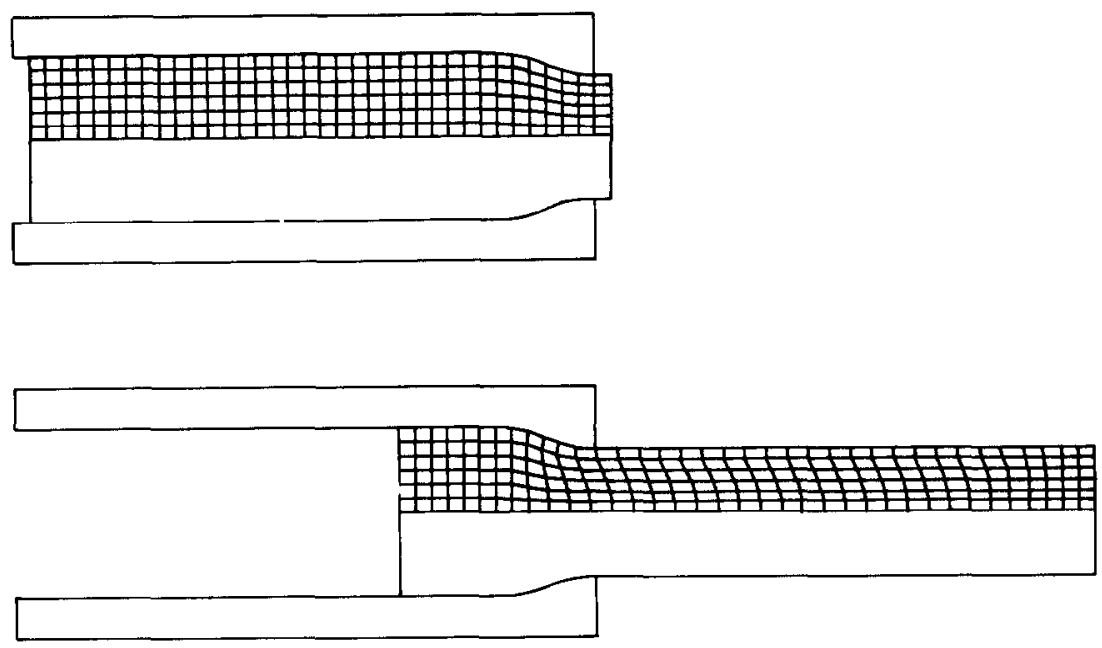

Fig. 2. The $6 \times 36$ quadrilateral finite element mesh shown before and after extrusion.

The steady state process, in which the extrusion has continued until an invariant pattern of deformation has developed relative to the die, is of major interest for applications since this is a common mode of process operation. For a convected mesh which initially consisted of a uniform distribution of undeformed elements, as shown in fig. 2 , such a steady state would generate a residual pattern of deformation in the extruded sheet which exhibits a translational invariance along its length except for transient regions adjacent to the free end of the sheet and in the neighborhood of the die. This is so since the material which has left the die is subjected to no surface tractions, and each congruent cross-section has been subjected to the same history of deformation followed by unloading to zero resultant load and zero traction on the lateral surfaces. In such a region the equations of equilibrium and the conditions of steady extrusion through symmetric dies prescribe that the residual stresses comprise a distribution of longitudinal stress $\sigma_{x x}(y)$ which is even in $y$ and self-equilibrating:

$$
\int_{0}^{b} \sigma_{x x}(y) \mathrm{d} y=0
$$

where $2 b$ is the thickness of the extrudate. For such a stress field, which exhibits translational invariance in the $x$ direction with zero surface tractions on $y= \pm b$, equilibrium demands that

$$
\sigma_{y y}=\sigma_{x y}=0 .
$$

This deduction assumes a restriction of the nature of St. Venant's principle in elasticity. The transient region adjacent to the free end involves the generation of shearing stresses to transform the longitudinal residual stress pattern $\sigma_{x x}(y)$ to zero surface traction on the free end.

The computer program used was a plane strain, elastic-plastic, finite element code, valid for small strains and employing constant strain triangle elements, modified in accordance with [3] and [4] in order to make it valid for large strains and to conform with the sequentially updated 
Lagrangian scheme described in section 2. By appropriately reinterpreting the original program. only two major changes were required: (1) introduction of initial stress stiffness terms, required by the variational principle, into the computation of each triangular element stiffness matrix contribution and (2) introduction of dilatation and rotation terms into the computations updating stress at the end of each increment in order to properly convert Jaumann increments of Kirchhoff stress into material increments of Cauchy stress.

Symmetry of the die about the center line of the billet permitted the solution to be carried out on half the billet only on one side of the center-line. This symmetry determined the boundary conditions on the center line to require zero shear stress and zero normal velocity. On the basis of the discussion of boundary conditions in the previous section, those on the center line will make no contribution to the surface integral of the variational principle since this boundary is straight. For the curved boundary at the metal-die interface, the contribution to the surface integral, e(1. (13), can most easily be introduced in terms of nodal forces acting on the boundary nodes. The surface integral contains the tangential component of the traction rate multiplied by $\delta v_{1}$, the tangential velocity variation. The incremental contribution of each nodal force at the boundary during the time $\Delta t$ is the product of the force magnitude, the angle between the normals after and before the increment of motion, and the variation $\delta v_{1}$. In order to make the boundary nodes follow the curved boundary accurately, a predicted displacement increment is used to establish the direction of the nodal velocity vector so that during each increment the boundary node follows a chord, and thus the average normal velocity is zero during an increment. The angles between the normals at the ends of the chord comprise the curvature term $\kappa v_{1} \Delta t$ in the surface integral.

At the exit from the die the boundary conditions change discontinuously from sliding contact against a rigid boundary to the condition of a traction-free surface. As a boundary node emerges from the reduction region the direction of the nodal velocity is no longer prescribed. Instead, during the period of time extending until the next boundary node emerges, we reduced the normal reaction force incrementally to zero, and thereafter maintained it at zero. The second boundary condition for each node is simply the zero shear traction condition.

In the numerical evaluation of the increments of stress and velocity in a time step $\Delta t$. such increments are in practice calculated directly by multiplying eq. (4) throughout by $\Delta t$. For each time step the stress and strain increments $\Delta \sigma$ and $\Delta \varepsilon$ for each of the constant strain elements used must follow the stress-strain relation. This causes some computational difficulty, particularly when plastic flow first sets in, since the gradient drops rapidly by a factor of order $10^{-3}$ from the elastic modulus to the work-hardening modulus. Initially the stress-strain point for each element moves up the elastic line and is so extrapolated for each time step. It thus leaves the stress-strain curve when the yield stress is exceeded. The computer program automatically selects time steps so that this initial departure from the stress-strain curve cannot exceed a preset limit, ty pically a $4 \%$ deviation in stress. In subsequent steps the direction of the stress increment-strain increment vector for each element is selected in such a way that the stress-strain point approdches the stress-strain curve while maintaining a good approximation to the incremental tangent modulus. In practice the initial deviation from the stress-strain curve approaches the above-mentioned limit for only one or two of the elements which are becoming plastic for the first time. The measured stress-strain curve is followed much more closely for most elements for almost all of the time. A similar difficulty occurs on unloading after plastic flow and reloading into the plastic region; however, this applies to very few elements. The time steps so selected are small so that about 20 time steps occur while a single element at the surface exits from the die. This fact has a bearing on the already discussed 
discontinuous change to a traction-free boundary condition as the extrudate exits from the die.

As mentioned in the first paragraph of section 2 , convection terms become particularly significant for materials with small strain-hardening coefficients. Although such terms are properly accounted for in the variational formulation, extra care must also be exercised in the selection of the finite element discretization. The difficulty arises as a result of the fact that a plastically flowing material becomes more nearly incompressible for small strain-hardening coefficients. In such situations the deformation degrees of freedom of many common finite elements are "used up" in satisfying the near-incompressibility constraint with the result that the aggregate of elements becomes "locked up" and acts more stiffly than the continuum it is supposed to represent. Of the several possible remedies for this problem described by Nagtegaal, Parks and Rice [5] we chose to adopt patterns of triangular elements which form quadrilaterals having straight diagonals. It is shown in [5] that when three of the four triangles forming such a quadrilateral satisfy the incompressibility condition, the fourth triangle does so automatically, and thus sufficient degrees of freedom become available to permit the variational principle to generate an accurate solution. Thus the finite element mesh utilized consisted of the quadrilaterals shown in fig. 2, each composed of four constant-strain triangles formed by its diagonals.

The incremental stiffness equations deduced from the variational principle, which must be solved for each incremental step, are linear algebraic equations with a symmetric coefficient matrix (the composite incremental stiffness matrix) which is banded. By appropriately ordering the unknown nodal velocities, the bandwidth is minimized and the number of operations required for solution is correspondingly reduced. To minimize computer storage requirements, a compressed storage scheme is used which simply involves storing only the nonzero diagonals of the lower triangular part of the incremental stiffncss matrix. A modified Cholesky solution procedure is used to solve the stiffness equations. Many modifications have been made in the program to improve efficiency, accuracy and flexibility. These range from the introduction of automatic finite element mesh and time increment step size generating procedures to the development of restart and data retrieval capabilities.

\section{Plane strain extrusion of an aluminum billet}

The stress and deformation fields for the extrusion of an aluminum billet were evaluated using the program already discussed. An aluminum slab of finite length in an initially stress-free state was forced into a symmetrical die after sliding between smooth rigid plates which simulated a cylinder in the analogous axisymmetric case. Fig. 2 shows the initial configuration in which the billet was assumed to have been machined to fit the die. Also shown is the configuration after most of the billet had been extruded through the die. A rigid smooth piston pressing against the rear face of the slab and moving with prescribed velocity provided the driving force. As mentioned in the previous section, each of the quadrilaterals shown in fig. 2 contained four triangular finite elements.

Isotropic work-hardening was assumed with the Mises yield condition. The hardening function was selected to fit a measured tensile stress-strain curve, corresponding to the stress-strain curve in octahedral shear shown in fig. 3. Some stress components in a metal-forming process such as extrusion can easily reach magnitudes of six times the yield stress in shear, which is seen to be almost equal to the hardening modulus, so that for correct analysis it is necessary to include convec- 


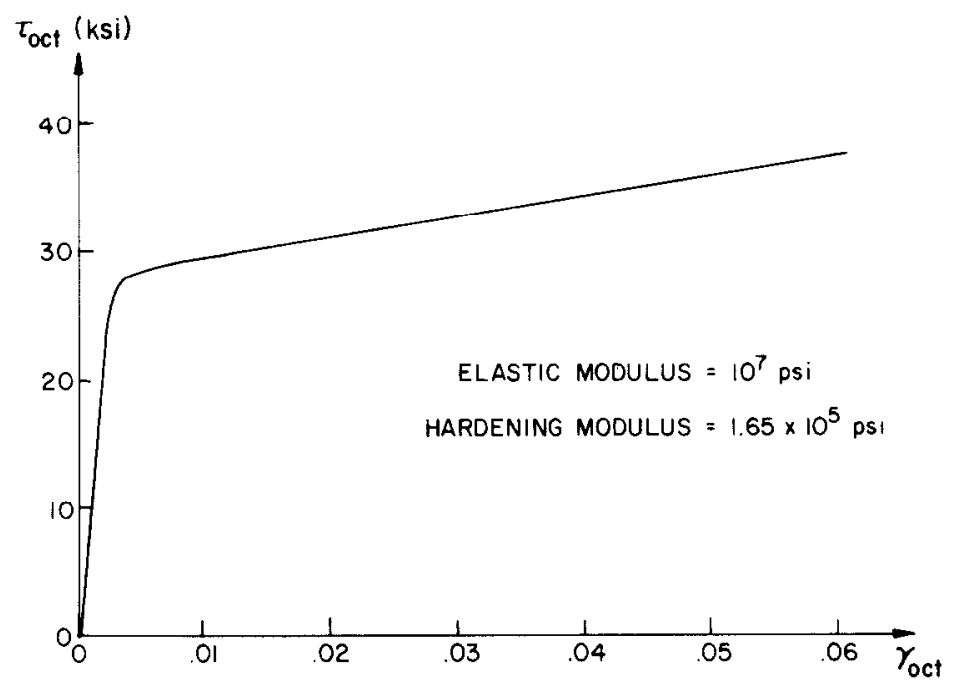

Fig. 3. The stress-strain relation in octahedral shear for an aluminum alloy.

tion terms as discussed in section 2 and as included in the program used.

The die considered in this study produced a $25 \%$ thickness reduction over a distance of $1.2 a$, where $a$ is half of the original sheet thickness, and was shaped in the form of a 5 th order polynomial curve with zero curvature and slope at both ends. A network of $6 \times 36=216$ quadrilateral elements was employed, and the resulting composite incremental stiffness matrix was symmetric, of order 518 and half-bandwidth 18, after elimination by static condensation of 432 degrees of freedom corresponding to quadrilateral centroid nodes. The extrusion calculation was continued until steady state had been achieved as indicated by the appearance of a uniform region of residual stress in the extrudate. The resulting total displacement of the driving piston was $5.59 a$ (in a computation requiring 522 incremental steps).

It may appear from fig. 2 that the extrudate emerges as a rigid body; nevertheless, unloading strains of the order of elastic strains do occur but are not apparent at the scale of that figure. However, they are crucial in generating the redistribution of stress leading to the distribution of uniform longitudinal residual stress discussed in the previous section. The plastic shear accompanying the section reduction associated with extrusion through the die is evident in the deformed configuration of fig. 2. This is associated with the "lost work" in this process since the shearing absorbs energy but does not contribute to the section reduction. The pattern of distortion of the finite element mesh shown in fig. 2 is clearly within acceptable limits for computational accuracy.

Fig. 4 shows the variation of driving force required per square inch of piston area. The curve with smaller oscillations, marked " $6 \times 36$ network", corresponds to the configuration described above and shown in fig. 2 , where there are six quadrilateral finite elements across the half-width of the slab. As will be described later, a coarser network was also considered with four elements across the half-width, which yields the curve with more pronounced oscillations.

It is clear from fig. 4 that apart from the oscillation the driving force settles down to a steady state value after a piston motion of just over the half-width of the slab, which is close to the die length of $1.2 a$. For the early displacement increments the deformation is primarily elastic. As 


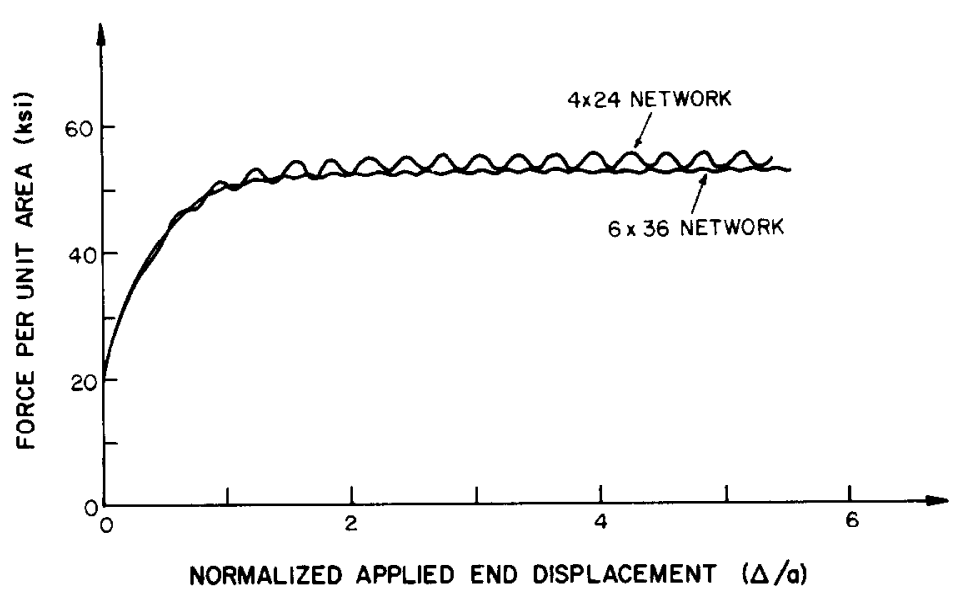

Fig. 4. The variation of driving force for the extrusion process.

plastic flow sets in, a more gradual increase in force occurs, which then settles down, on the average, to a steady state value. The oscillation is associated with the fact that the curved boundary is approximated as the polygonal line formed by the straight edges of the triangular finite elements adjacent to the boundary, but since the elements move, the polygonal approximation varies. In fact the polygonal line assumes the same shape each time the finite element mesh advances by one mesh spacing - this explains the periodicity and establishes the wave length of the oscillation. Although the oscillation is much more pronounced for the coarser mesh, the average force magnitude agrees very well with the result for the finer mesh - this suggests that the latter provides a satisfactory model for the continuum.

The fact that the driving force does settle quickly to a steady state value indicates that the procedure of using patterns of triangular elements which form quadrilaterals with diagonals - termed crossed-triangles - does overcome the constraint of near-incompressibility at large plastic flow as discussed by Nagtegaal, Parks and Rice [5]. Experience with other patterns of triangular elements indicates that with their use the deduced driving force would have continued to increase with displacement.

Fig. 5 shows the extent of the active plastic elements in the steady state mode after a displacement of $4.56 a$, which corresponds to the deformed state in fig. 2 . The current plastic flow is essentially limited to a single region. The separated plastic triangles in the extrudate are essentially hovering on the borderline of unloading, and one or two of them may load and unload several times before settling into the unloaded condition. Thus a single triangle of four in a quadrilateral, separated from the continuous plastic region, exhibits current plasticity; this fact is associated with the use of the crossed triangles. There can be an appreciable discrepancy in the magnitudes of stress components in the four triangles, although the average values yield a smooth stress distribution in the work-piece. The yield stress values in the separate triangles are much closer however. In plotting stress distributions we use averages for the quadrilaterals.

Fig. 6 gives a plot of the distribution of longitudinal stress components-each curve represents the stress variation along a line passing through a different row of 36 quadrilateral centroids. The initial distance of the row from the center line, labelled "lateral station", is indicated for each stress curve. The region between $x=8.5 a$ and $x=11 a$ is clearly a region in which the longitudinal 


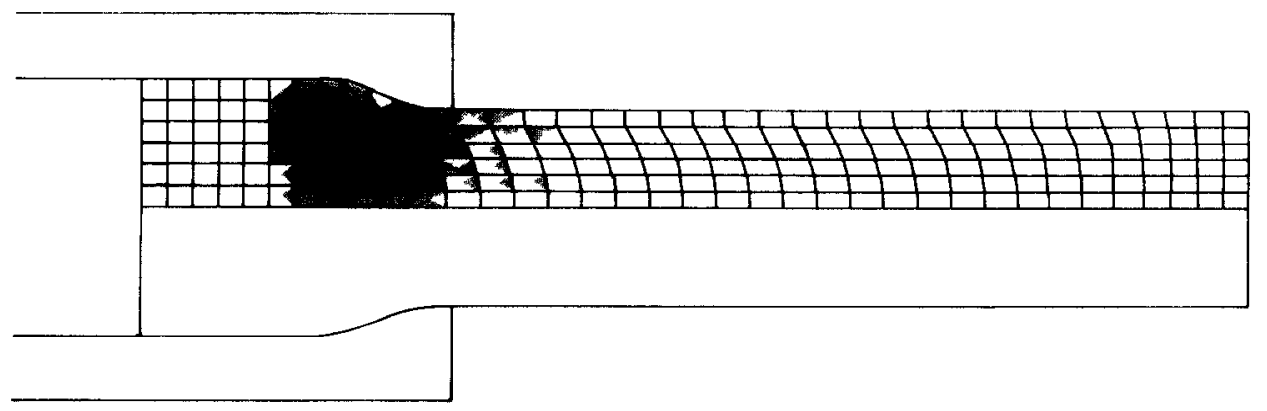

Fig. 5. The configuration of the plastic region in the steady state.

stress has no longitudinal variation and thus represents a steady state residual stress pattern. The longitudinal stress does exhibit a lateral variation in this region which ranges from compression at the midplane to tension at the free lateral surfaces.

The state of stress in the uniform residual stress region was discussed in the previous section, where it was shown (15) that the lateral stress $\sigma_{y y}$ and the shear stress $\sigma_{x y}$ are zero there due to the requirements of equilibrium. As a check on the calculation fig. 7 shows the distribution of shear stress along the same rows of quadrilateral centroids. Virtually zero shear does occur for all rows in the residual stress region. The shear distribution required to transform the residual stress

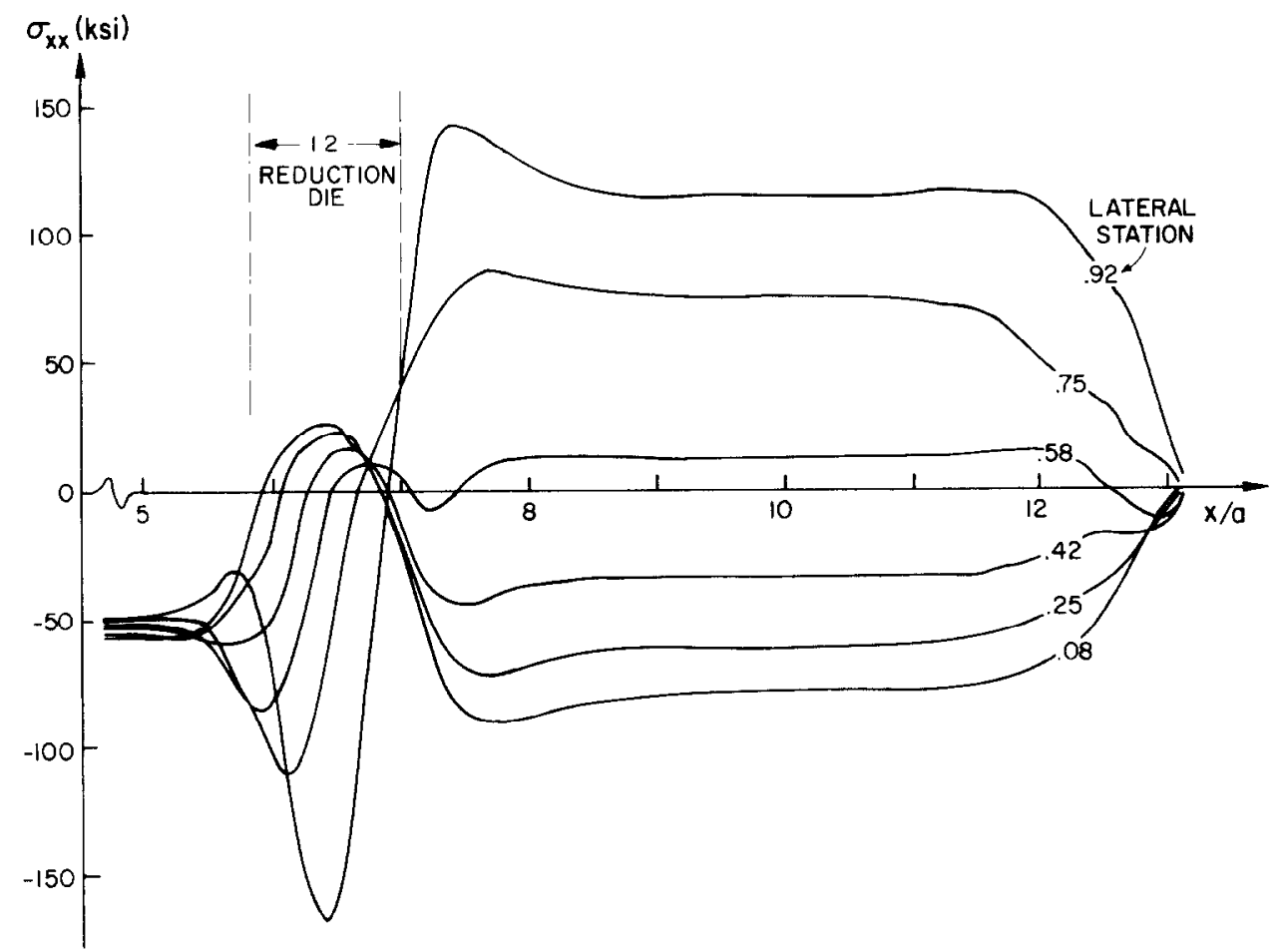

Fig. 6. The distribution of longitudinal stress $\sigma_{x x}$ at steady state. 


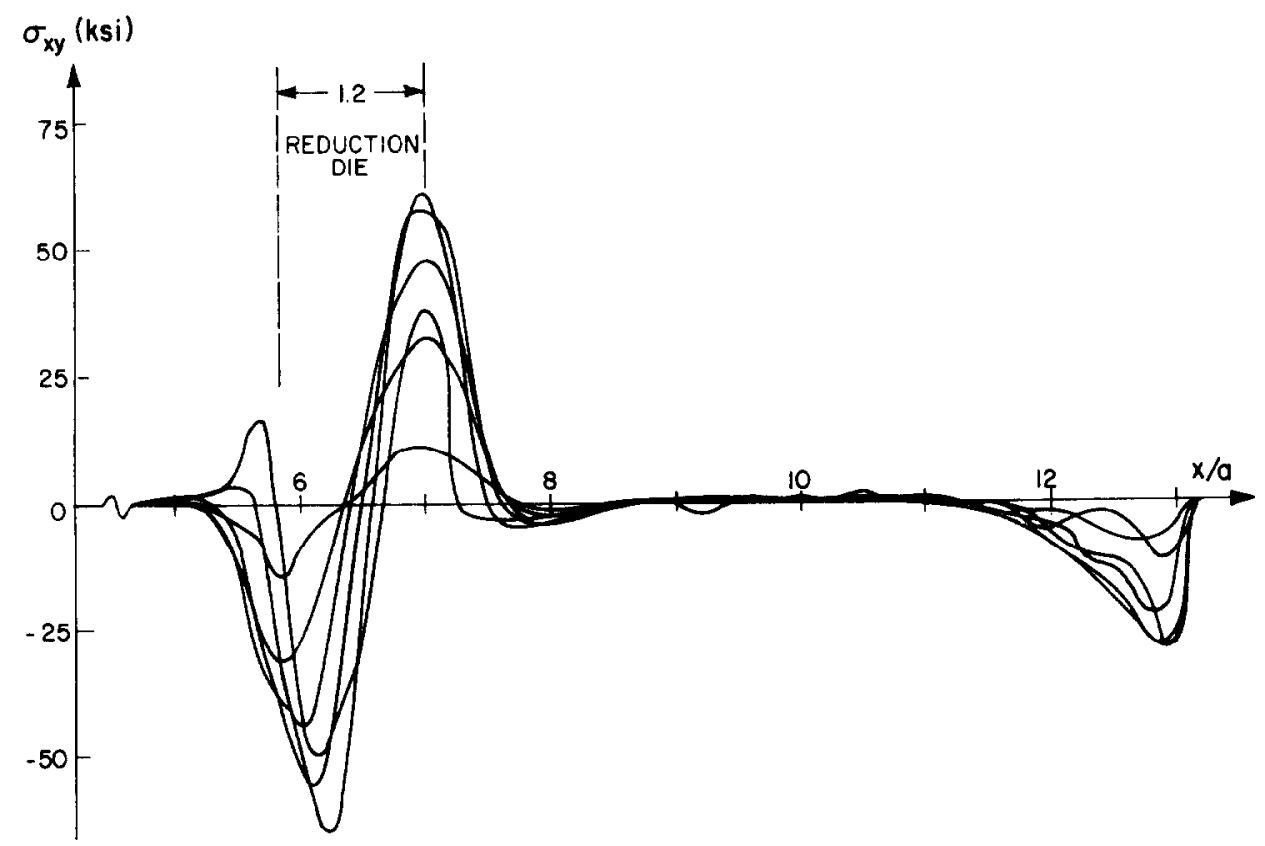

Fig. 7. The distribution of shear stress $\sigma_{x y}$ at steady state.

pattern to zero surface traction at the free end is evident for $x>11 a$ as well as the large shear components at emergence from the die.

The stress distribution is determined from the variational principle (4) for the velocity distribution. The latter gives the strain-rate history from (2), and the stress is then determined from the elastic-plastic constitutive relation. Some inaccuracy in the stresses can therefore be expected, and a good check is to test for equilibrium. In the residual stress region the resultant longitudinal traction across a section is zero since no tractions are applied to the extruded material. That no traction is transmitted across the free surface at the forward end of the extrudate can be checked by summing the nodal forces there, and this sum per unit area is down by a factor of $10^{-7}$ from the residual stress levels. Integrating the residual stress levels across the section by Simpson's rule yields an average stress of magnitude $5 \%$ of the maximum residual stress. This result suggests that the accuracy of the stress determination is of this order.

Features of the stress distribution which are of technological interest can be seen in fig. 6. For example, the tensile peak which occurs at the surfaces shortly after emergence from the die (at $x=7.2 a$ ) corresponds to an extrusion defect sometimes observed, namely the appearance of surface cracks. Modification of die geometry at the exit can eliminate this problem, and it might be possible to investigate this condition numerically with the present program. Tensile stresses along the center-line inside the die are also a potentially significant feature.

As mentioned in connection with the driving force variation in fig. 4, the solution was evaluated for a coarser finite element mesh of four quadrilaterals across the half-width of the entering billet. The corresponding longitudinal stress distributions are shown in fig. 8 . These are similar to the more accurate results in fig. 6 and show the main features exhibited there. For quantitative comparison fig. 9 shows the distribution of residual stress across the section taken from the two sets of results. They exhibit the same general trend with the coarser network determining stresses 


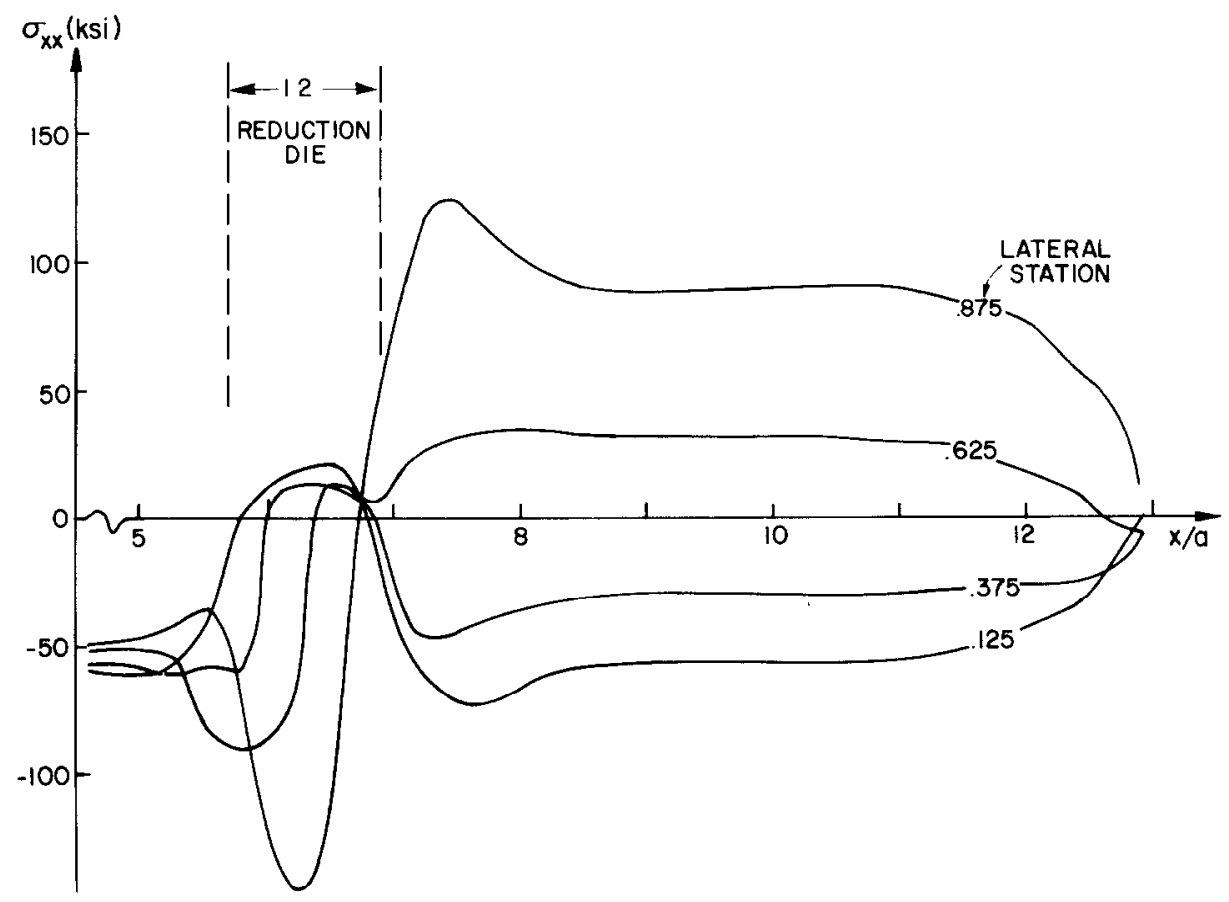

Fig. 8. The distribution of longitudinal stress deduced from a coarser finite element network.

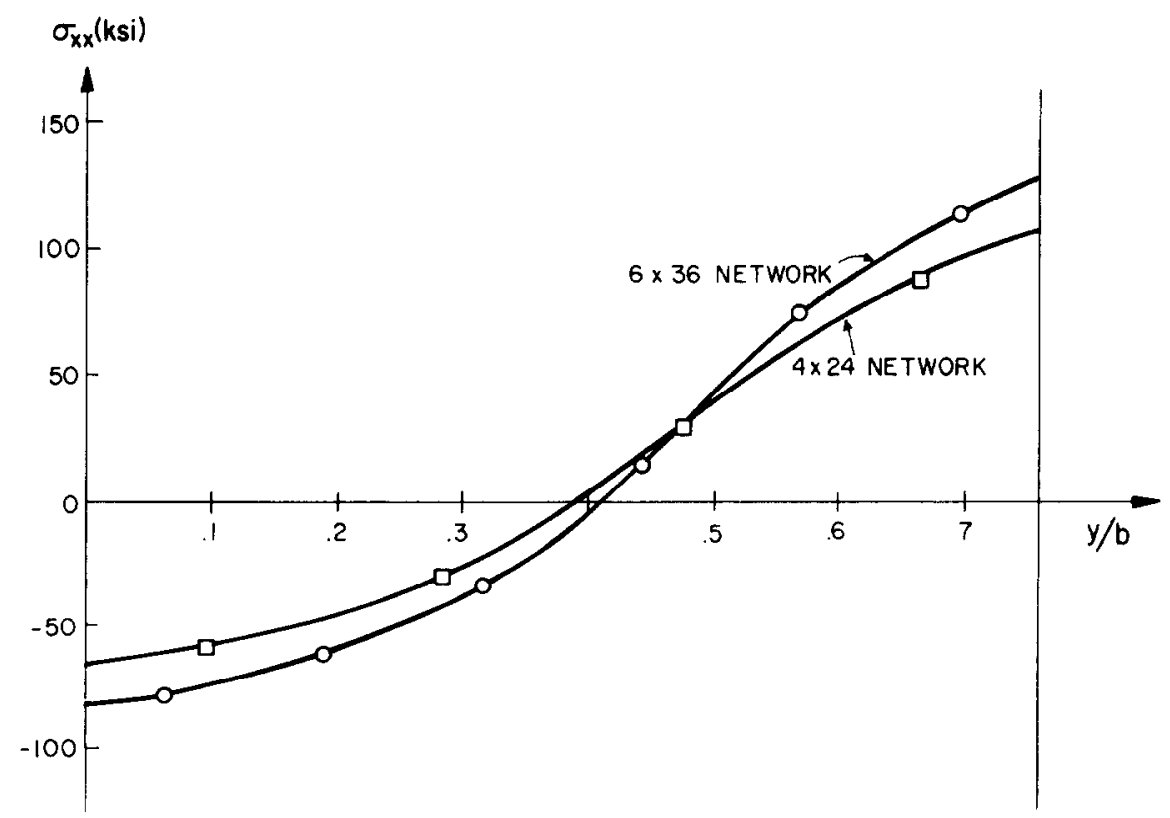

Fig. 9. Comparison of residual stress distributions using two finite element networks. 
reduced by somewhat less than $20 \%$. This could be consistent with the $5 \%$ error suggested by checking equilibrium for the more accurate solution.

The die contour considered in the calculation was determined by a fifth order polynomial with zero curvature at each end. This provided a smooth variation of curvature which gave a smooth variation of the boundary traction term (13). However, in a localized region at the die entrance, tensile tractions were deduced across the billet-die interface. This indicates that separation would occur and that the traction would remain zero until contact was re-established. We plan to modify the program to permit separation, but since this is a highly localized change in traction, we do not expect it to alter appreciably the rest of the solution; hence, local tensile tractions were permitted in the present solution but restricted to the immediate neighborhood of the die entrance.

Although the variation of only two stress components has been illustrated in this paper, the solution provides complete information on the history of the stress distribution throughout the process. The stress history in a material element tends to be quite complicated and far removed from proportional loading and unloading. This has a bearing on the adequacy of the elastic-plastic constitutive relation that is used. In spite of the complexity of the solution the care exercised in devising an efficient numerical solution procedure and computer program led to a running time of only about 15 minutes on the IBM 370/168 computer at the Stanford Center for Information Processing.

\section{Discussion}

The solution we have presented comes at the beginning of a project to analyze metal-forming processes. It thus comprises a simple first attempt on such problems, and it is expected that more general boundary conditions and constitutive relations will be incorporated and also schemes to reduce the time and hence cost of computation. For example, if analysis of the steady state solution is of primary interest, the selection of an initial stress field much closer to the final field than the unstressed state might lead to appreciable computational savings. Direct attack on the steady state case might also be possible without the need to evaluate the transient solution as in the present approach. On the other hand, more involved constitutive laws might be necessary which will complicate the computational problem. For example, adoption of an isotropic work-hardening law does not permit the inclusion of a Bauschinger effect. Since unloading and reverse loading often occur when the work-piece leaves the metal-forming region of the equipment, the maintained elastic stiffness of the body under reversed loading probably leads to excessive residual-stress levels. In some cases it may be important to reduce this source of error.

\section{References}

[1] J.R. Rice, A note on the "small strain" formulation for elastic-plastic problems, Tech. Rep. N00014-67-A-0191-0003/8, Div. Eng., Brown Univ. (1970).

[2] R. Hill, Some basic principles in the mechanics of solids without a natural time, J. Mech. Phys. Solids 7 (1959) $209-225$.

[3] R.M. McMeeking and J.R. Rice, Finite-element formulations for problems of large elastic-plastic deformation, Int. J. Solids Structs. 11 (1975) $601-616$.

[4] E.H. Lee, The basis for an elastic-plastic code, SUDAM Report No. 76-1, Div. Appl. Mech., Dep. Mech. Eng., Stanford Univ. (May 1976) to appear in: Metal forming plasticity (Springer Verlag through the Centre International des Sciences Mecaniques).

[5] J.C. Nagtegaal, D.M. Parks and J.R. Rice, On numerically accurate finite element solutions in the fully plastic range, Comp. Meths. Appl. Mech. Eng. 4 (1974) 153-177. 